\title{
A Survey of Routing Protocols of Wireless Sensor Networks
}

\author{
Zhe Yang and Abbas Mohammed \\ Blekinge Institute of Technology \\ Sweden
}

\section{Introduction}

Networked micro-sensor technology is a key technology for the future. It has been identified as one of the most important technologies for the 21st century and is regarded to revolutionize information gathering and processing in applications (Heinzelman et al., 1999). Advances in Micro-Electro-Mechanical Systems (MEMS) and low-power integrated digital electronics have inspired the development of micro-sensors (Sohrabi, 2000). Such sensors are generally equipped with date processing, communication, and information collecting capabilities. They can detect the variation of ambient conditions in the environment surrounding the sensors and transform them into electric signals. Interests in sensor networks have motivated intensive research in the past few years emphasizing the potential of cooperations among sensors in date collecting and processing, coordination and management of the sensing activity, and date flow to the sink. Wireless Sensor Networks (WSNs) is one of the architecture of sensor networks. WSN can be formed by sensors in an ad-hoc manner. It belongs to the general family of sensor networks that use multiple distributed sensors to collect information on entities of interest.

As in many technologies, research on sensor networks was originally motivated by military applications. Early research was done by military using sensor networks for defence dealing with events at contiguous levels. Around 1980 modern research on sensor networks started with the distributed sensor networks program at the US Defense Advanced Research Projects Agency (DARPA). During this period universities and institutes did an intensive research in technology components for sensor networks about designing acoustic sensors, protocols to link processes of working on a common application in a network, self-location algorithms, distributed software and developing test beds. In 1990s there was an important shift of sensor network research due to advances in computing and communications. Small size, low cost sensors are designed to be based upon MEMS technology, wireless networking and low power processors, which make sensors possible to be deployed in a wireless fashion. The shift has led and influenced the latest research on networking and information processing techniques of sensor networks.

\subsection{Communication Architecture and Applications of WSNs}

A typical WSN contains a large number of sensor nodes, which send collected data via radio transmitter to a sink. The decrease in both the size and the cost due to the development of MEMS has led to smart disposable micro sensors, which can be networked through wireless 
connections to the Internet. Fig. 1 shows an architecture of communications in a WSN. Sensor nodes are capable of organizing themselves, and collect information about the phenomenon and route data via neighbouring sensors to the sink. The gateway in Fig. 1 could be a fixed or mobile node with an ability of connecting sensor networks to the outer existing communication infrastructure, such as Internet, cellular and satellite networks.

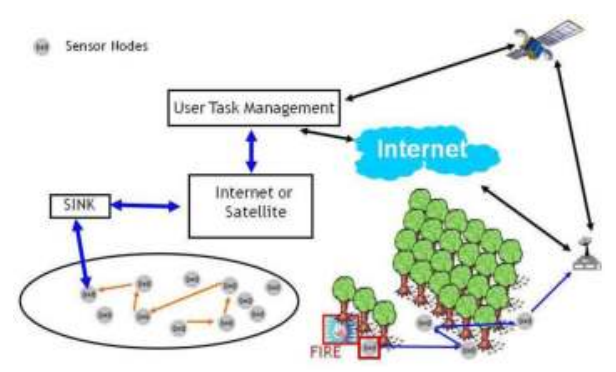

Fig. 1. Communication architecture of a WSN

Depending on applications to reveal some characteristics about phenomena in the area, sensor nodes can be deployed on the ground, in the air, under water, on bodies, in vehicles and inside buildings (Akyildiz et al., 2002; Xu, 2002). Publications and current applications have shown these connected sensor nodes have the potential in both consumer and military applications, e.g. target field imaging, intrusion detection, weather monitoring, security and tactical surveillance, distributed computing, traffic and inventory control, detecting ambient conditions such as temperature, humidity, movement, sound and light. Deployment of these sensor nodes can be in a random fashion like dropping from a helicopter in a disaster management applicaiton for environment survelience, or manually(Akyildiz et al., 2002).

\subsection{Network Layer in WSNs}

Sensor nodes are constrained by energy supply and bandwidth. Such constraints combined with the deployment of a large number of nodes are challenges to the design and maintenance of the network. Energy-awareness needs to be considered at all layers of a protocol stack. Physical and data link layers, which are generally common for all kind of applications, also need to consider these limitations. Thus research on these layers have focused on radio communication hardware, energy-aware medium access control (MAC) protocols (Demirkol et al., 2006; Intel, 2004). Table 1 gives a full view of protocol stack for communications in sensor networks.

The main aim at the networking layer is to find the route to transmit data from sensor nodes to the sink in an energy-efficient and reliable manner in order to maximally extend the lifetime of the network. Routings in sensor networks are challenging due to several characteristics distinguishing from established wireless communication networks in following areas.

1. It is not possible to build a global addressing scheme for a large number of sensors deployed. The addressing scheme, e.g. Internet Protocol (IP), needs to maintain routing tables for the global topology. Updating in a dynamic environment of a typical sensor network's application leads to heavy overhead in terms of time, memory and energy. Therefore a classical IP-based protocol is not applicable to sensor networks (Akyildiz et al., 2002). 


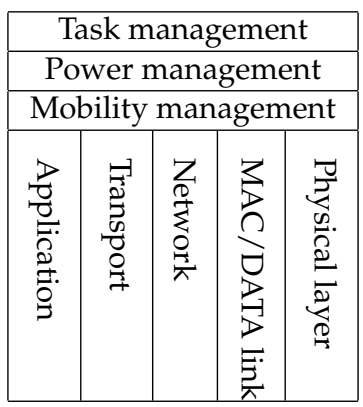

Table 1. Protocol stack for communications in sensor network

2. Compared to a typical communication network, e.g. mobile communication networks, almost all applications of sensor networks require the flow of sensed data from different sources to the same sink (Akyildiz et al., 2002). Most prevalent wireless networks today, e.g. cellular network, are based on cells which are regions divided geographically. A mobile terminal in a cell only communicates with the base station serving the cell. A peer-to-peer communication between two mobile terminals doesn't exist. Communications are established through different base stations. However sensor nodes in WSNs send data to the sink based on a multiple hop routing composed by distributed networking and control functions in sensor nodes.

3. Data traffic generated by sensor nodes have significant redundancy because multiple sensors with a similar distance to the phenomena may generate the same data. Such redundancy needs to be eliminated by using proper routing protocols to improve energy and bandwidth utilization.

4. Different resource management protocols in the stack have to consider constrains of sensor node in terms of its transmission power, residual energy, processing and storage capacity.

Many specific algorithms have been proposed to solve these problems of routing data in sensor networks (Niculescu, 2005). These routing mechanisms have to consider characteristics of sensor nodes and application requirements. Classically most routing protocols could be classified as data-centric, hierarchical and location based protocols depending on the network structure and applications. A few distinct protocols are based on network flow and quality of service (QoS) awareness.

Date-centric protocols are query-based and depend on the naming of data of interest, which could help to reduce repeated transmissions.

Hierarchical protocols use the cluster concept in the network to divide sensors into different clusters and choose cluster heads to aggregate and reduce transmission of data in order to save energy.

Location-based protocols utilize the position information to relay data to the destination.

The chapter is organized as follows: In the rest of section 1 , we will briefly summarize the design issues for sensor networks on data routing. In section 2, 3, 4 and 5, different routing approaches of current reserach will be presented. In section 6 information of research platforms, simulation, and development tools of WSNs will be introduced. Conclusions and future work will be given in section 7 . 


\subsection{Design Factors of WSNs}

Due to the large number of sensor nodes and the dynamics of their operating environment, it poses unique challenges in the architecture design of sensor networks. Routing design is closely related to the system architecture mode. In this section, we will summarize architectural issues of sensor networks and how they affect routing process in WSNs.

Dynamic Network: Basically a WSN consists of three components: sensor node, sink and event. Sensor nodes and sink are assumed to be fixed or mobile. Currently sensor nodes in most applications are assumed to be stationary, but it is still necessary to support the mobility of sinks or gateways in the network. Thus the stability of routing data is an important design factor, in addition to energy consumptions and bandwidth utilizations (Akyildiz et al., 2002). Node Deployment: The topology of node deployment is application dependent and affects the performance of the routing protocol. If the nodes are deployed randomly, they need to create an infrastructure in an ad-hoc manner and organize themselves to establish paths to route the data. If nodes are deployed manually with pre-arranged locations, pre-determined path could be built to route data (Akyildiz et al., 2002). In addition, the position of the sink or gateway is also important to optimize routing paths.

Energy Constrains: The process of setting up the routes in the network is greatly affected by energy considerations. Since radio transmission degrades with distance much faster than transmission in free space, it implies that communication distance and energy consumption must be well managed. Directed routing would perform well enough if all sensor nodes are close to the sink. However most of the time it is necessary to use multiple hop routing to consume less power than directive routing, because sensors are usually randomly scattered in the area. However this introduces significant overhead for topology management and MAC protocols.

Data Transmission and Dissemination Models: Based on applications of sensor networks, the data delivery to the sink can be continuous, event-driven, query-driven or hybrid (Akyildiz et al., 2002; Demirkol et al., 2006). In the continuous model, each sensor node sends data periodically. While in the event-driven and a query-driven applications, sensor node only start to transmit data when the event occurs or a query is generated by the sink. Some applications combine continuous, even-driven and query-driven data delivery. Corresponding to the transmission model of data delivery mentioned above, data flow transmitted between sensor nodes can be classified as: broadcast, unicast and multicast subject to different routing protocols. Performance of using protocols is application dependent. For example, broadcasting can generate high overhead, but it is suitable for dynamic changes in the topology of the network. A major advantage of broadcast is the lack of a complex network layer protocol for routing, address and location management. Existing sensor network efforts have mostly relied on this approach.

MAC Protocol Design: To operate a wireless sensor network successfully, MAC protocols are important networking issues, which need to consider energy consumption and complexity of implementation. Numerous energy-efficient MAC protocols have been developed for sensor networks, such as S-MAC (Ye et al., 2002) and T-MAC (Dan \& Langendoen, 2003), but these protocols still operate in an address-based fashion, which rely on message passing to individual neighbours. A data-centric based MAC protocol is investigated in (Ditzel \& Langendoen, 2005) to follow a data-centric routing. Nodes access the communication resources following an active-sleep regime, alleviating the problem of idle-listening.

There are other issues, such as coverage area, scalability, transmission media, which could also affect the design and performance of routing protocol. 


\section{Date-centric Protocols}

Due to the dense deployment and dynamic environment of sensor nodes in many applications, it is not possible to assign global identifiers to each node (Akyildiz et al., 2002). Random deployment and dynamics make it hard to select a specific set of sensor nodes to be queried. Thus routing in the system should operate autonomously, changing its configuration as required. This means protocols are able to select a set of sensor nodes and can employ data aggregation during the delivery while considering energy consumption. Connections can not be planned in advance but should emerge on-demand. However, in traditional address-based networks, routings are created between nodes and managed in the network layer of the communication stack.

Users would be more interested in querying a specific area rather than a single node because of a large population of sensor nodes. In data-centric routing, the sink requests information from nodes in certain area and waits for responses from sensors located in the selected area. To facilitate date-centric characteristics of sensor queries, an attribute-based naming scheme is used to specify the properties of data. Each node involved in the transmission plays the same role.

\subsection{Flooding and Gossiping}

Flooding and Gossiping: These two classical mechanisms to deliver data in sensor networks don't need any routing algorithms. In a flooding mechanism, each sensor receives a data packet and then broadcasts to all neighbouring nodes. When the packet arrives at the destination or the maximum number of hops is reached, the process of broadcasting is stopped. It is easy to implement flooding but with several drawbacks like implosion, which could be caused by sending duplicated messages to the same node, overlapping when two nodes sense in the same region and send similar data to its neighbours (Heinzelman et al., 1999). Energyawareness is not considered in these mechanisms. Gossiping solves the problem of implosion by sending information to a random neighbour instead of a classical broadcasting mechanism sending packets to all neighbours. However gossiping causes another problem - delay in a propagation of data among sensor nodes.

\subsection{SPIN}

Sensor Protocols for Information via Negotiation (SPIN): SPIN is an outcome of an early research in a data-centric routing mechanism. The main idea in SPIN is to use meta data instead of a full data packet transmitted at each node to all nodes (Heinzelman et al., 1999). It assumes that nodes in close proximity have similar data. Before transmission, data collected by nodes are exchanged among sensors via data advertisement mechanism, which enable nodes to distribute data which other nodes don't pose. Negotiation process between neighboured nodes are performed by naming the data using high-level descriptors before any data start to be transmitted (Akyildiz et al., 2002). SPIN ensures that low redundant data sent throughout the network and solve problems, such as wasting energy and bandwidth to send extra copies of data by sensors in the same area (Akyildiz et al., 2002), of a broadcasting mechanism, e.g. flooding. Each sensor node can operate more efficiently and conserve energy by sending data after negotiation instead of sending all data. However data collecting mechanism cannot guarantee the delivery of data. For example, if the node of interest is far away from the sensing source and nodes between the source and destination are not interested in that data, such data can not be be routed to the destination. Two types of SPIN have been developed. SPIN-1 doesn't deal with energy efficiency and SPIN-2 is energy aware. 
SPIN is more efficient than the protocol based on flooding and has relatively quick convergence in terms of latency. The type of protocols can be used for both mobile or stationary events. The negotiation process is fairly simple. The main drawback of the protocol is the energy consumption caused by idle nodes being always active.

\subsection{Directed Diffusion}

Directed Diffusion The protocol is an important breakthrough in a data-centric routing research of sensor networks. The idea behind diffusing data through sensor nodes aims to use a scheme of naming data for all communications. It uses attribute-value pairs for the data and queries the sensors on demand. By defining an interest through a list of attribute-value pairs, such as name of objects, interval, duration, geographical area etc., it can create a query to communicate with nodes. Data is cached at intermediate nodes for aggregation and loop prevention. Interests are propagated by unicast, multicast or broadcast from the sink to nodes. Once sensor nodes collect information, they compare with the data in the interest pre-stored in the cache and respond to specified interest. The local gradient is set by propagating interest from sink to source, where a path reinforcement between a source and sink can be realized by resending interest messages frequently. The data sent back to a sink by unicast and multicast consists of the data rate, duration and expiration time derived from the received interest. Path repairs in directed diffusion could also be possible by employing multiple paths in advance (Sohrabi, 2000). Thus if one path fails, an alternative is chosen to replace it.

A directed diffusion protocol consumes much less energy by having less traffic compared to flooding. It uses the best path based on local gradient to have a good latency bound. A retransmission of interest makes the protocol robust. The drawback of the protocol is that directed diffusion is application dependent, because it is based on a query-driven data delivery model. If the application, like environment monitoring, requires continuous transmission to the sink, it will not work effectively with a query-driven on demand data model (Akyildiz et al., 2002). Comparing the data with the pre-stored interests will also generate redundant overhead at the sensors. A retramission or an alternative path maintenance is needed.

\subsection{Other Data-centric Protocols}

SPIN and direct diffusion have motivated designs of other data-centric protocols. Energyaware routing, rumor routing, gradient-based routing and Constrained Anisotropic Diffusion Routing (CADR) follow a similar concept of using queries to certain regions to get response (Akyildiz et al., 2002; Braninsky \& Estrin, 2002; Dan \& Langendoen, 2003; Heinzelman et al., 1999; Niculescu, 2005).

\section{Hierarchical Protocols}

The dense and random deployment of nodes causes the scalability to be one of the design issues in sensor networks. Usually a network with a single gateway is not scalable for a larger set of sensors since sensors are not capable of extended communication period. Networking different clusters is proposed to allow system to cope with an additional load and a large coverage area with a long-haul communication.

The main aim of a hierarchical routing is to maintain the energy consumption of sensor nodes. A cluster formation in a multi-hop sensor network is typically based on the energy reserve of sensors and their distances to the cluster head (Akyildiz et al., 2002). The cluster head with a higher-energy node, can be used to process and send the information. In addition, the rest of sensors in that cluster can perform tasks of sensing. 


\subsection{LEACH}

Low-Energy Adaptive Clustering Hierarchy (LEACH): The idea of LEACH algorithm is to form clusters of the sensor nodes based on the received signal strength, and use local cluster heads as routers to the sink (Heinzelman et al., 2000). This routing mechanism saves energy since the transmissions are mainly managed by cluster heads. Initially cluster heads are randomly selected and changed over time in order to spread load and balance the energy dispersions of nodes. A cluster head compresses data arriving from nodes belonging to its cluster and sends an aggregated packet to the sink. Adaptive clustering is employed to increase the lifetime of the system. LEACH assumes that each node has enough power to transmit signals to reach cluster head and has equal computational power to work in different MAC protocols. Thus it is not applicable to deploy in large regions due to the variation of distances between sensors and head of clusters (Akyildiz et al., 2002). Moreover the idea of dynamic clustering brings extra overhead, such as rotation of cluster head, advertisement etc., and accordingly consumes energy.

LEACH uses Code Division Multiple Access (CDMA) or Time Division Multiple Access (TDMA) MAC to share channels (Akyildiz et al., 2002; Heinzelman et al., 2000). These MAC protocols are widely used in modern cellular communication systems. By scheduling nodes into different sub-channels by codes or time slots, they can avoid interference between each other. So traffic in sensor network is largely collision-free, which saves energy compared to MAC protocols such as Carrier Sense Multiple Access (CSMA) (Ye \& Heidemann, 2003). However adopting these scheduled MAC schemes causes idle listening, which happens when the radio is listening to the channel before transmitting possible data. Due to constantly listening to the channel, the cost of energy is especially high in many sensor network applications where no data is transmitted during the period of no reported event. Idle listening is a dominant factor of radio energy consumption (Demirkol et al., 2006).

LEACH optimizes energy by shutting down radios of sensor nodes and load balancing. The scalability can be reached by a distributed hierarchical approach. It is easy to aggregate data at the head of a cluster and send to a user or sink. However disadvantages of LEACH are related to its hierarchical formation, where the failure and selection of the cluster head is a problem and difficult to optimize. It is also expensive to assume that all nodes are capable of communicating over an extended distance.

\subsection{GPSR}

Greedy Perimeter Stateless Routing (GPSR): The GPSR is a routing protocol to transfer the data packets in wireless datagram networks. GPSR is based on an algorithm which combines Greedy Packet Forwarding and Perimeter Forwarding methods. Greedy Packet forwarding is a strategy enabling the source to know the geographic position of the destination integrated in the route request. GPSR provides each node of the network with a local table to list identifies and positions of neighbouring nodes. A proactive broadcast refreshes the table of each node in a regular time interval. The source node gives the packet a destination address. This address will not be changed by any node which forwards the packet.

GPSR performs better than dynamic source routing and only needs local information for packet forwarding by using greedy forwarding strategy. The protocol can be operated by using energy-efficient MAC protocols to increase the energy efficiency. 


\subsection{Other Hierarchical Protocols}

LEACH inspires many hierarchical protocols such as Power-Efficient Gathering in Sensor Information System (PEGASIS), Threshold sensitive Energy Efficient sensor Network protocol (TEEN), Adaptive TEEN (APTEEN) etc..

PEGASIS is an enhanced protocol using CDMA capable nodes over LEACH to extend network lifetime by using only one node in a chain to transmit to the sink instead of multiple nodes. TEEN and APTEEN (Zhao et al., 2006) are based on time-critical applications using TDMA schedule. They are developed to be responsive to sudden changes, which require the precision of time in non-period and periodic reports such as the temperature in the sensed area in a disaster management application.

\section{Location-based Protocols}

Since sensor nodes are mostly spatially and randomly scattered in an area, there is no addressing scheme, e.g. IP-addresses, for sensor network. In most applications, location information is needed in order to know the separating distance between particular nodes and optimize routing in an energy efficient way. Relative coordinates can be built by exchanging distances between neighbour nodes in order to approximate the strength of incoming signals. Alternatively equipping low-power global positioning system (GPS) devices into hardware of sensor nodes can obtain location information directly through communications with satellites. While global coordinates and compatibility are desirable, the GPS may not always be used because of line-of-sight conditions, power requirement and other limits (Niculescu, 2005). In some applications information of the sensor area is known, so using locations of sensors can build a query directly diffused only to region of interests, and decrease the number of transmission significantly.

\subsection{MECN and SMECN}

Minimum Energy Communication Network (MECN): By using low power GPS devices, sensor nodes in MECN can setup and maintain a minimum energy network ( $\mathrm{Xu}, 2002)$. MECN assumes a master-node as the information sink and develops a minimum power topology for each node. MECN identifies a relay region for each node, which is consisted of nodes in a surrounding area where transmitting through those nodes is more energy-efficient than direct transmission. A sub-network build in MECN is based on having less number of nodes which can consume less power for transmission between any two specific nodes. In this way, global minimum power paths are found without considering all the nodes in the network. Optimal links are calculated based on the position coordinates updated by using GPS. Moreover it can dynamically adapt to elimination of nodes or the deployment of new sensors since it is capable of self-reconfiguring.

Small MECN (SMECN): It is assumed that in MECH each node can transmit to others, which are not possible in all cases if there are obstacles between any pair of considered nodes. SMECN is proposed to cope with obstacles. Although it is still assumed in SMECN that node could be fully connected, the sub-network established in SMECN for minimum energy is smaller in terms of the number of edges compared with the one in $\mathrm{MECH}$ if broadcasts are able to reach all nodes in a circular region around the broadcaster. Thus the number of hops in SMECH will decrease, therefore energy can be saved. More overhead happens when finding a sub-network with a smaller number of edges in SMECN $(\mathrm{Xu}, 2002)$. 


\subsection{GAF and GEAR}

Geographic Adaptive Fidelity (GAF): It is a GPS location-based routing algorithm designed primarily for mobile ad-hoc network (Akyildiz et al., 2002). The idea of the protocol is to setup a virtual grid based on location information and conserve energy by turning off some nodes depending on the redundancy in the network without affecting the system fidelity to extend the network lifetime. GAF may also be considered as a hierarchical protocol, where the clusters are based on geographic locations. A representative node is selected in each particular cluster to transmit the data to other nodes. GAF performs at least as well as a normal ad-hoc routing protocol, e.g. dynamic source routing, but with substantial conservation of energy, which is realized by the protocol to tune for parameters like estimated node active time, time for node discovery and status being active and sleep.

Geographic and Energy Aware Routing (GEAR): Estimating separation distance is an alternative to use location information from GPS. GEAR uses of geographic information and relays queries to certain regions because data queries contain geographic attributes. The main idea is to restrict the number of interests in directed diffusion by only considering a certain region rather than sending the interests to the whole network, thus it conserves energy and improves the lifetime of network.

\section{Network Flow and QoS-based Protocols}

There are some effective routing protocols proposed in different approaches which don't fit the above classification. In network flow, route is modeled and solved in a network flow. QoS-based protocols consider end-to-end delay requirements and establish paths in sensor networks. A few examples of these are discussed in this section.

Maximum Life Energy Routing (MLER): It is proposed in (Chang \& Tassiulas, 2000) as a solution to the problem of routing in sensor networks based on a network flow approach. The main idea of this approach is to maximally extend the network lifetime by defining link cost as a function of residual energy of node, and the require transmission energy using that link. By maximizing the lifetime of the network, the protocol leads to establish traffic distribution, which is a possible solution to the routing problem in sensor networks.

Sequential Assignment Routing (SAR): SAR is the first protocol for WSN that includes a notion of QoS. The objective of the SAR algorithms is to minimize the average weighted QoS metric throughout the lifetime of the network (Akyildiz et al., 2002). It creates trees rooted at one-hop neighbours of the sink by taking QoS metric, energy resource on each path and priority level of each packet into consideration. Through creating trees, SAR built multiple paths from sink to sensors. It ensures the fault-tolerance and easy recovery. However when huge sensors are deployed, SAR will suffer from the overhead of maintaining the table and states at each sensor nodes.

Different QoS-aware MAC protocols have also been proposed for WSNs. Reinforcement Learning based MAC (RL-MAC) (Liu \& Elhanany, 2006) is an novel adaptive MAC for WSNs, which employs a reinforcement learning framework. Nodes actively infer the state of other nodes, using a reinforcement learning based control mechanism. QoS is easily implemented in this proposed framework. QoS-aware MAC (Q-MAC) in (Liu et al., 2005) is another innovative idea, which minimizes the energy consumption in multi-hop WSNs and provides QoS by differentiating network services based on priority level. It allows sensor networks to reflect on the criticality of data packets originating from the different sensor nodes. 


\section{Research Platforms and Tools}

Great interests have motivated intensive research to realize the vision of WSN in the past few years. Research prototype sensor nodes (UCB motes, WINS, Smart Dust, PC104, etc.) are designed and manufactured. Simulation and development tools are also being developed.

\subsection{Sensor platforms}

1. MICA motes

MICA mote is a commercially available product that has been used widely by researchers and developers. MICA motes use an Atmel Atmega 128L microcontroller to provide bidirectional communication at $50 \mathrm{kbps}$ and a pair of $A A$ batteries to provide energy. The operation system (OS) cooperating with the MICA is called the TinyOS , which is currently widely used.

2. Rockwell WINS

Rockwell WINS uses a StrongARM $1100 \mathrm{CPU}$ running at $133 \mathrm{MHz}, 1 \mathrm{MB}$ of FLASH memory, $1 \mathrm{MB}$ of RAM, a $100 \mathrm{kbps}$ radio, and has to operate on two $9 \mathrm{~V}$ batteries. This is considered to be towards the high end of sensor network devices.

3. Smart Dust

Tiny nodes, called Smart Dust, are densely deployed to float in the air and organize themselves into a sensor network to achieve a surveillance task. It has more strict constraint with energy consumption and a simply undivided architecture. Currently sensor networks are considered to evolve toward this small dust if technological advance permits such miniaturization and copes with other existing limits (Hollick et al., 2004).

4. PC-104 based nodes

Nodes based on PC-104 are much larger than Mica motes. They are widely used as parent nodes in hierarchical sensor networks. The PC-104 based testbed is mainly funded by DARPA SenseIT program. It is built upon off-the-shelf PC-104 based products. Each node has an AMD ElanSC400 CPU,16MB RAM and 16MB IDE Flash Disk.

\subsection{Simulation and development tools}

1. UCB tools chain for development in Motes

The tool chains are composed of four parts: TinyOS kernel, NesC compiler, TOSSIM simulator and TinyDB processing system. TinyOS has a component-based programming model, codified by the NesC language. TinyOS is not an OS in the traditional sense; it is a programming framework for embedded systems and set of components that enable building an application-specific OS into each application.

2. NS-2 and Nam NS-2 is developed since 1985 by collaborations of USC Berkeley, USC/ISI, MIT, C-Mellon, Sun, DARPA and NSF (Shih et al., 2001). It is suitable for simulating wireless sensor network operations. Nam is a package for visualization using for NS-2.

3. Sensor Sim is a simulation framework for modeling sensor networks built upon the NS-2 simulator and provides additional feature for modeling sensor networks. 


\section{Conclusions and Future Work}

Routing technologies in sensor networks have attracted considerable attention in recent years. They are subject to challenges which are different to traditional networks. In general, a routing protocol needs to deal with scalability, energy efficiency, robustness, latency, low computation and memory usage. In this survey, we have summarized typical research results on routings protocols in sensor networks and classified them into different classes as data-centric, hierarchical and location-based. Examples of network flow and QoS modeling methods, which follows other approaches, have also been discussed.

Data-centric protocols name the data and query the nodes based on attributes of the data. The most important aspect of this paradigm is the content of the sensor-generated data, which drives most implementations of the upper layers: discovery, routing, and querying (Niculescu, 2005). Research follow this paradigm in order to avoid the overhead of forming clusters. On the other hand, the naming scheme is not sufficient for complex queries and is not easily extended to cover a larger area.

Cluster-based routing protocols divide sensor nodes into different groups to efficiently relay the sensed data to the sink. A cluster head performs aggregation and fusion of data and sends data directly to the sink on behalf of other member nodes. It gives solutions to the problem of the formation of clusters and optimization of the energy consumption. The process of the communication between the head sensor is an open issue for reserach.

Protocols employing location information and topological deployment of sensor nodes are classified as location based ones. There is no need for routing tables in this network since each node can decide how to relay packets based on the destination to the packet and some local information about its immediate neighbours. However since it is the source must know the position of the destination, it is still an implicit requirement in many applications. Moreover how to aid energy efficient routing by using the local geometrical information is still a problem.

Most research protocols pay main attentions to the energy efficiency without addressing many issues like QoS. QoS-aware routings in sensor networks have many applications like real time targeting, emergent event triggering in monitoring applications etc. Current research is aiming at controlling QoS requirement in an energy-efficient application environment. Common issues like routing around obstacles, scalabilities, adaptive applications etc. are open for designs of protocols.

Sensor network is a popular research area and has applications in the real world. Protocols present today have their own set of problems which need to be improved. Most protocols dealing with energy efficiency can be significantly improved with robustness and scalability. Most results are empirical nowadays and more theoretical work can be done to incorporate game theory for modeling. Simulation tools can also be improved by focusing on sensor network in mind.

\section{References}

Akyildiz, I. F., Su, W., Sankarasubramaniam, Y. \& Cayirci, E. (2002). A survey on sensor network, IEEE Communications Magazine .

Braninsky, D. \& Estrin, D. (2002). Rumor routing algorithm for sensor networks, First Workshop on Sensor Networks and Applications (WSNA), Atlanta,GA. 
Chang, J. H. \& Tassiulas, L. (2000). Maximum lifetime routing in wireless sensor networks, The Advanced Telecommunications and Information Distriution Research Program (ATIRP'2000), College Park,MD.

Dan, T. V. \& Langendoen, K. (2003). An adaptive energy-efficient mac protocol for wireless sensor networks, the first international conference on Embedded Networked Sensor Systems, ACM press, pp. 171-180.

Demirkol, I., Ersoy, C. \& Alagoz, F. (2006). Mac protocols for wireless sensor networks: a survey, Communications Magazine, IEEE 44(4): 115-121.

Ditzel, M. \& Langendoen, K. (2005). D3: Data-centric data dissemination in wireless sensor networks, Wireless Technology 2005 pp. Page(s):185 - 188.

Heinzelman, W., Chandrakasan, A. \& Balakrishnan, H. (2000). Energy-efficient communication protocol for wireless sensor networks, the Hawaii International Conference System Sciences, Hawaii.

Heinzelman, W., Kulik, J. \& Balakrishnan, H. (1999). Adaptive protocols for information dissemination in wireless sensor networks, the 5th Annual ACM/IEEE International of the First Workshop on Sensor Networks and Applications (WSNA), Altlanta, GA, USA.

Hollick, M., Martinovic, I., Krop, T. \& Rimac, I. (2004). A survey on dependable routing in sensor networks, ad hoc networks, and cellular networks, the 30th EUROMICRO Conference.

Intel (2004). Instrumenting the word-an introduction to wireless sensor networks.

Liu, Y., Elhanany, I. \& Qi, H. (2005). An energy-efficient qos-aware media access control protocol for wireless sensor networks, IEEE MASS 2005.

Liu, Z. \& Elhanany, I. (2006). Rl-mac: A qos-aware reinforcement learning based mac protocol for wireless sensor networks, 2006 IEEE International Conference on Networking, Sensing and Control 2006, ICNSC '06, pp. 768 - 773.

Niculescu, D. (2005). Communication paradigms for sensor networks, IEEE Communication Magazine .

Shih, E., Calhoun, B. H., Cho, S. H. \& Chandrakasan, A. P. (2001). Energy-efficient link layer for wireless microsensor networks, IEEE 2001 Computer Society Workshop on VLSI, pp. 16-21.

Sohrabi, K. (2000). Protocols for self-organization of a wireless sensor network, IEEE Personal Communications 7(5): 16-27.

$\mathrm{Xu}, \mathrm{N}$. (2002). A survey of sensor network applications, IEEE Communications Magazine $4 \mathbf{0 .}$

Ye, W. \& Heidemann, J. (2003). Medium access control in wireless sensor networks, Technical Report ISI-TR-580.

Ye, W., Heidemann, J. \& Estrin, D. (2002). An energy efficient mac protocol for wireless sensor networks, 21st conference of the IEEE Computer and Communications Societies (INFOCOM) 11(1): 1567-1576.

Zhao, L., Xu, C. N., Xu, Y. J. \& Li, X. W. (2006). Energy-aware qos control for wireless sensor network, 2006 1st IEEE Conference on Industrial Electronics and Applications, Vols 1-3, pp. 1663-1668. 


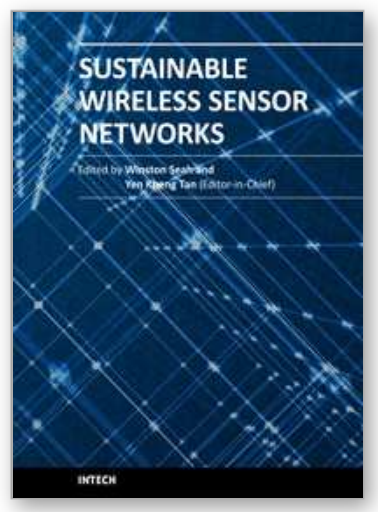

\author{
Sustainable Wireless Sensor Networks \\ Edited by Yen Kheng Tan
}

ISBN 978-953-307-297-5

Hard cover, 574 pages

Publisher InTech

Published online 14, December, 2010

Published in print edition December, 2010

Wireless Sensor Networks came into prominence around the start of this millennium motivated by the omnipresent scenario of small-sized sensors with limited power deployed in large numbers over an area to monitor different phenomenon. The sole motivation of a large portion of research efforts has been to maximize the lifetime of the network, where network lifetime is typically measured from the instant of deployment to the point when one of the nodes has expended its limited power source and becomes in-operational â $€^{\prime \prime}$ commonly referred as first node failure. Over the years, research has increasingly adopted ideas from wireless communications as well as embedded systems development in order to move this technology closer to realistic deployment scenarios. In such a rich research area as wireless sensor networks, it is difficult if not impossible to provide a comprehensive coverage of all relevant aspects. In this book, we hope to give the reader with a snapshot of some aspects of wireless sensor networks research that provides both a high level overview as well as detailed discussion on specific areas.

\title{
How to reference
}

In order to correctly reference this scholarly work, feel free to copy and paste the following:

Abbas Mohammed and Zhe Yang (2010). A Survey on Routing Protocols for Wireless Sensor Networks, Sustainable Wireless Sensor Networks, Yen Kheng Tan (Ed.), ISBN: 978-953-307-297-5, InTech, Available from: http://www.intechopen.com/books/sustainable-wireless-sensor-networks/a-survey-on-routing-protocolsfor-wireless-sensor-networks

\section{INTECH}

open science | open minds

\section{InTech Europe}

University Campus STeP Ri

Slavka Krautzeka 83/A

51000 Rijeka, Croatia

Phone: +385 (51) 770447

Fax: +385 (51) 686166

www.intechopen.com

\section{InTech China}

Unit 405, Office Block, Hotel Equatorial Shanghai

No.65, Yan An Road (West), Shanghai, 200040, China

中国上海市延安西路65号上海国际贵都大饭店办公楼 405 单元

Phone: +86-21-62489820

Fax: +86-21-62489821 
(C) 2010 The Author(s). Licensee IntechOpen. This chapter is distributed under the terms of the Creative Commons Attribution-NonCommercialShareAlike-3.0 License, which permits use, distribution and reproduction for non-commercial purposes, provided the original is properly cited and derivative works building on this content are distributed under the same license. 